\title{
Apropiación de las tecnologías de información y comunicación en el ámbito educativo venezolano
}

\author{
Appropriation of Information and Communication Technologies in \\ The Venezuelan Education
}

\author{
Ingrith Roselys Marcano-Rojas ${ }^{1}$ \\ Universidad de Oriente, \\ Núcleo Nueva Esparta \\ Isla de Margarita, estado Nueva Esparta, Venezuela \\ ingrith.marcano@ne.udo.edu.ve
}

Recibido: 7 junio 2013 Aceptado: 4 diciembre 2014 Corregido: 26 diciembre 2014

\begin{abstract}
Resumen: El propósito de esta investigación es valorar el uso de la tecnología de la información y la comunicación como medio de apoyo a la formación docente en el ámbito venezolano, precisando cómo ha sido la intervención tecnológica en el entorno educativo. Se trata de una revisión documental donde se referencian experiencias de actualización docente emprendidas en países como Panamá, Colombia, México y Argentina, y se comparan con los esfuerzos realizados en Venezuela. Destacando que se han creado planes para mejorar la calidad de la formación docente en materia de Informática; así como proyectos dedicados a la atención de las comunidades en el uso del computador y de las comunicaciones, ejemplo: dotación de laboratorios informáticos, portales Web dedicados a la educación, el Plan Nacional de Alfabetización Tecnológica (PNAT), entre otros. Se concluye que se pueden concentrar esfuerzos para coadyuvar al fortalecimiento de la formación docente, y aprovechar el potencial que tiene la Nación para el apoyo de sus planes tecnológicos, considerando así, al contingente de estudiantes de educación universitaria que deben prestar servicio comunitario como requisito para optar al título profesional. En correspondencia con esto, se propone emprender un trabajo significativo con los estudiantes voluntarios del servicio comunitario para que aporten sus conocimientos en la preparación de los docentes en formación, y constituir una propuesta en pro de fortalecer el trabajo colaborativo entre Instituciones educativas.
\end{abstract}

Palabras clave: TIC, Curso de Formación, Oportunidad Educacional, Tecnología Educacional.

Abstract: The purpose of this research is to evaluate the use of information and communication technology as a means to support teacher training in Venezuela, specifying how the technological intervention has been taken in the educational environment. This is a documentary work that makes

1 Doctora en Educación de la Universidad Pedagógica Experimental Libertador (UPEL-Venezuela). Actualmente labora como Docente adscrita a la Carrera Licenciatura en Informática en la Universidad de Oriente (UDO-Venezuela), Núcleo de Nueva Esparta (UDONE). Es Licenciada en Informática (egresada de la UDONE) y Magister en Educación, Mención Gerencia Educacional (egresada de la UPEL). Fue Coordinadora de la Carrera Licenciatura en Informática de la UDONE durante el periodo comprendido entre Abril de 2010 y Julio de 2012. Es Investigadora adscrita a la Comisión de Investigación de la Universidad de Oriente, está acreditada como Investigador Tipo A en el Programa de Estímulo al Investigador e Innovador Nacional (PEII 2011-2013). Ha participado como ponente en congresos nacionales e internacionales, como el IX Congreso Científico de la Universidad de Oriente celebrado en Cumaná-Venezuela en Noviembre 2012, y en el I Congreso Internacional de Ingeniería Informática y Sistemas de Información CIIISI 2012, organizado en el marco de la XVI Convención Científica de Ingeniería y Arquitectura celebrado en Cuba 2012. Entre sus publicaciones recientes se destacan: Creación de un Método de Análisis de Problemas Algorítmicos en Revista Espacios Digital, entre otras. 
mention to experiences of some countries such as Panama, Colombia, Mexico and Argentina in relation to the use of ICT in education, comparing them with national effort, emphasizing out that in Venezuela, the resources have been earmarked, and some projects have been created in order to improve the quality of education, especially in the field of information and communication technologies, likewise, many projects have emerged from the national level, that are directed to the attention of general community to computer use and communications, for this reason, projects like the National Plan for Technological Literacy (PNAT), and Web portals of distance learning were created. Finally, it concludes that the efforts should be concentrated to contribute the fortification of teacher training in our country, taking into account the great potential that the nation has to support its technology plans, considering the number of students in higher education who are willing to serve communities in their educational area. So that, it is time to undertake a meaningful work with volunteer students from community service, in order to share their knowledge in the preparation of teachers in training or in exercise, being this a proposal in favor of strengthening collaborative work between educational institutions.

Keywords: Information and Communication Technology (ICT), training courses, educational opportunities, educational technology.

\section{Las tecnologías de información y comunicación (TIC)}

Las tecnologías de la información y la comunicación se han convertido en elementos esenciales que impulsan cambios positivos en la economía y la sociedad mundial; es así como el acceso a la información digital se ha considerado tarea elemental para quienes quieren evolucionar en sus formas de vida y por ende, de trabajo. Las TIC pueden ser definidas como un conjunto de elementos tecnológicos que aporta la Informática para que sean utilizados por el ser humano, en aprovechamiento de los programas de computadora, los dispositivos electrónicos, así como de las tecnologías de la telecomunicación; todo ello, conjugado para ser implementado como una herramienta poderosa que generará utilidad siempre y cuando sea usado adecuadamente.

En ese mismo sentido, el Programa de las Naciones Unidas para el Desarrollo (PNUD, 2002), en el Informe sobre Desarrollo Humano en Venezuela, expresa lo siguiente:

Las TIC se conciben como el universo de dos conjuntos, representados por las tradicionales Tecnologías de la Comunicación (TC) - constituidas principalmente por la radio, la televisión y la telefonía convencional - y por las Tecnologías de la información (TI) caracterizadas por la digitalización de las tecnologías de registros de contenidos (informática, de las comunicaciones (telemática) y de las interfases (mediática), lo cual ha sido posible dada la adaptabilidad que ofrece la computación.(p. 10)

Por su parte, Miratia (2005), haciendo referencia a los trabajos de Garcias (1996) y Cabero (1996), sostiene que "Las TIC se agrupan en tres grandes sistemas de comunicación: el video, la informática y la telecomunicación“ (p. 12); y en respuesta a su interrogante ¿qué medios o instrumentos forman parte de esto? responde:

Abarcan los siguiente medios: el video interactivo, el videotexto, el teletexto, la televisión por cable y satélite, la web con sus hiperdocumentos, el CD ROM, los sistemas multimedia, la teleconferencia en sus distintos formatos (audio conferencia, video conferencia, 
conferencia audiográfica, conferencia por computadora y teleconferencia desktop), los sistemas expertos, la realidad virtual, la telemática y la telepresencia. (p. 12)

Ambas definiciones concuerdan en los elementos representativos de las TIC, sin embargo, no hacen señalamiento a la finalidad o razón de ser de éstas, que no es más que proveer herramientas de trabajo al ser humano, por cuanto éste ha sido su creador, impulsado por sus propias necesidades.

Desde la segunda mitad del siglo XX, la expansión en el uso de las computadoras, así como de otros dispositivos electrónicos marcó la pauta para quienes tuvieron el privilegio de incorporar esa tecnología en su quehacer diario, lo que mejoró su forma de vida dado que pudo alcanzar mayor efectividad en su trabajo. En paralelo a esta realidad, se encontraban las personas e instituciones que servían de simples espectadores ante el uso de la tecnología sin tener la oportunidad de apropiarse de ésta para alcanzar mejoras en su desempeño. Es así como nos abordan las innovaciones; primero son accesibles para grupos privilegiados según su estatus social, laboral, su avance tecnológico, etcétera. Luego su uso se expande hacia otras comunidades de usuarios con características que, en comparación con los primeros, son limitadas ante el desconocimiento de éstas, y que, luego de conocerlas las comienzan a incorporar en búsqueda de los beneficios que ofrecen.

En este sentido, ya han pasado unos cuantos años desde que se inició la propagación de las TIC y el uso de Internet en el hogar, en la empresa, en las universidades, escuelas, liceos, así como en cualquier otro tipo de organización pública, comunitaria o privada. Con ello se abrieron las oportunidades de expansión de las empresas especializadas en el desarrollo del software, promoviendo la automatización de procesos de todo tipo, se desarrollaron los sistemas de información automatizados, se expandió el uso de las redes de comunicación; se considera una etapa de crecimiento y aprovechamiento del hardware y el software, los cuales han sido identificados en el PNUD (2002), como Infoestructura e Infocultura, respectivamente, a propósito de precisar la importancia que tiene el balance entre estos términos para el aprovechamiento adecuado de las TIC, así como para el desarrollo humano.

En este orden de ideas, la Infoestructura es esencial, pero no suficiente por sí sola, pues se requiere del desarrollo de los aspectos del estrato cultural para que se garantice su efectividad. Entonces, la incorporación de las TIC en el desarrollo humano requiere del desarrollo paralelo y balanceado de la infoestructura e infocultura (p. 47). Dicho trabajo requiere la intervención del Estado el cual ha dedicado un gran esfuerzo en pro de alcanzar la soberanía tecnológica, la inclusión al uso de las TIC y la incorporación de éstas en la gobernabilidad, así como en la educación.

$\mathrm{Al}$ respecto, las autoridades venezolanas con competencia en el plano tecnológico, tal como el Ministerio del Poder Popular de Ciencia, Tecnología e Industrias Intermedias, han llevado a ejecución un sinnúmero de estrategias y proyectos innovadores, a fin de coadyuvar a la apropiación de la tecnología, facilitando la disponibilidad de Infoestructura. Es así como se han realizado inversiones tecnológicas importantes, ejemplo de ello es el ensamblaje, en nuestro país, de computadoras marca: VIT, SIRAGON y E-TECH, y construcción de teléfonos celulares, cámaras, antenas satelitales, destacando como noticia de mayor 
trascendencia el lanzamiento de dos (2) satélites; el primero, VENESAT-1, mejor conocido como satélite Simón Bolívar, lanzado en octubre de 2008, a través del cual se fortalecen los servicios de telecomunicaciones, enalteciendo la soberanía tecnológica; el segundo, VRSS1 o satélite Miranda, lanzado en septiembre de 2012, destinado a la observación remota para el fotografiado de nuestra geografía, utilizado para apoyar a la toma de decisiones que involucren información espacial. Esta información es punto de referencia entre los adelantos que efectivamente el Estado está realizando, en procura de una importante inversión tecnológica; en ese sentido, se deben consolidar esfuerzos por parte de todos los venezolanos para aprovechar adecuadamente tales recursos, desarrollando una cultura apegada al logro de la democratización del uso de las TIC.

\section{Las TIC en la educación venezolana}

En el seno del Ministerio del Poder Popular para la Educación se han impulsado proyectos en pro de encaminar y garantizar el acceso a las TIC, entre los cuales destacan:

1. La Fundación Bolivariana de Informática y Telemática (FUNDABIT), a través de la cual se promueve la formación en el uso de las herramientas tecnológicas en el proceso educativo nacional. De este organismo se desprenden proyectos tales como: (a) los Centros Bolivarianos de Informática y Telemática (CBIT) creados para garantizar el acceso universal a la información, la democratización de las TIC y promover el desarrollo de la infocultura en todo el territorio venezolano; (b) CBIT-Móvil, gestado con el mismo propósito de los CBIT, dispone de los equipos en vehículos especiales que puedan llegar a las comunidades de difícil acceso a las telecomunicaciones en el ámbito nacional; vale destacar que estas unidades están a disposición de todas las zonas educativas; (c) Sistemas Fotovoltaicos, llevan paneles solares a los sitios más remotos donde no hay electricidad, de forma tal que los equipos puedan ser implementados en aquellas comunidades menos favorecidas, ofreciéndoles corriente eléctrica y por consiguiente acceso a las TIC; (d) Soluciones Educativas Tecnológicas (CPSET), que consisten en una serie de herramientas informáticas (softwares educativos, videos educativos, micros de radio, actividades de aprendizaje computarizadas, estrategias Web, entre otros), las cuales, mediante la participación interactiva con el computador, originan en los estudiantes la producción de conocimientos significativos dentro de un ambiente dinámico y colaborativo; mientras que para los docentes constituyen una herramienta valiosa al momento de preparar en forma motivadora y atractiva sus recursos pedagógicos; (e) Simoncitos, centros de educación inicial dotados con un computador por ambiente educativo, orientados a promover el desarrollo integral de los niños y niñas hasta los 6 años de edad o hasta su ingreso al primer grado de educación básica; y (f) Superaulas, espacios tecnológicos que han sido instalados en todo el país en un trabajo conjunto entre Fundabit y Cantv; éstas cuentan con once (11) computadores dotados software de contenido educativo, permitiendo además, el acceso a Internet de alta velocidad (Fundabit, s.f.).

2. La Red Nacional de Actualización Docente Mediante el Uso Educativo de la Informática y la telemática (RENADIT), es una red que reúne diferente organismos relacionados 
con la formación del docente venezolano, a fin de articular esfuerzos y recursos orientados a mejorar la práctica pedagógica en las diversas áreas del conocimiento con el uso pedagógico de las tecnologías. Este proyecto beneficia directamente a docentes de cualquier área y nivel educativo, profesionales e investigadores en el área de las TIC, incentiva la investigación y contribuye a las actividades de extensión y desarrollo de las diferentes líneas de exploración educativa en el país. (Cardona, 2004. p. 5).

3. Proyecto Canaima Educativo, nace con el objetivo de potenciar los aprendizajes en las y los estudiantes del Subsistema de Educación Básica mediante el uso de las portátiles Canaima como un recurso en constante actualización. (Ministerio del Poder Popular para la Educación, s.f.). A partir del 2013, amplió su cobertura al subsistema de educación media. A través de este proyecto se entregan las denominadas portátiles Canaimitas a estudiantes de las instituciones públicas nacionales, estatales, municipales, autónomas, también son otorgadas en aquellas instituciones privadas subsidiadas por el Estado.

En virtud de lo expuesto, se puede afirmar que actualmente las instituciones educativas están dotadas de laboratorios de computación donde, indistintamente del nivel educativo en curso, los estudiantes interactúan con el computador para conocerlo y utilizarlo, al menos en lo más elemental de su esencia.

Curiosamente, aún cuando estamos conscientes de esa realidad, nos encontramos en el transcurrir del tiempo con personas que a la luz de hoy día no utilizan el computador para su provecho personal o profesional, sin importar cual sea su área de acción. Es así como aún se pueden observar docentes que no tienen acceso al computador (sea en el colegio o en el hogar) para investigar sobre un tema de interés o llevar control del desempeño de sus estudiantes. Sobre la base de este planteamiento, surgen dos (2) interrogantes que invitan a reflexionar:

¿Los laboratorios de computación, así como los contenidos educativos que se imparten en ellos, son aprovechados completamente?, o es que no se ha logrado consolidar una cultura tecnológica que pueda ser comprendida, asumida y acomodada según las necesidades particulares de cada persona.

¿Por qué los estudiantes de nuevo ingreso al sistema de educación universitaria, generalmente, dan evidencia de fallas conceptuales de lo que es la computadora y de cuáles son las prestaciones que nos ofrecen estos artefactos? será que la infoestructura y la infocultura no han sido articuladas al servicio de los escolares para prepararse y aprender a explorar y obtener información de provecho utilizando las TIC.

En función de estas interrogantes, se debe puntualizar que la efectividad del aprendizaje dependerá, en buen porcentaje, de la calidad de la formación académica que se imparta. Es por ello que se deben concentrar esfuerzos para determinar en la actualidad cómo es la formación inicial del docente en relación con el uso de la tecnología. Esto dado que, en la era del conocimiento, es imprescindible que nuestros docentes se puedan desenvolver en el uso de las TIC, utilizándolas como herramienta de trabajo y aprovechando significativamente todas las bondades que éstas ofrecen y ponen al alcance de todos. Según Henríquez (2004), existe en Venezuela una preocupación por ofrecer al docente que está en preparación académica, una formación didáctico-educativa frente a la meramente técnica, donde se contemple la competencia en el manejo de los dispositivos de la computadora; también en la programación 
básica; el uso operativo del aparato (alfabetización en procesadores de texto, hoja de cálculo, etcétera); así como en evaluación de software; en los métodos de instrucción con computadores; desarrollo de destrezas como liderazgo de discusiones; promoción a la resolución de problemas; y selección de materiales (p. 64). Es decir, los contenidos utilizados en las asignaturas donde el futuro docente estudia el computador, deben someterse a una revisión a profundidad para comprobar su efectividad.

De la misma fuente se desprende la necesidad de intervenir en todos los componentes de la formación docente, porque no se trata solamente de que el profesorado adquiera conocimientos sobre las TIC que garanticen la actualidad de su acervo científico, sino también de que el profesor incorpore estas herramientas a su cultura profesional, impregne su práctica pedagógica. Es decir, debe experimentar un proceso de acomodación de esos conocimientos.

Estamos frente a un gran reto, el cual se refiere a lograr que nuestros docentes, tanto a nivel de educación primaria como secundaria, se formen adecuadamente en el uso de las TIC, de forma tal que puedan dar el ejemplo sobre el buen aprovechamiento de la tecnología, así como ofrecer orientaciones en esta materia a sus estudiantes; esto en virtud de que los niños y jóvenes de la actualidad, han crecido en medio de la expansión tecnológica y por ende, han percibido la influencia del computador y la Informática en la sociedad. Es así como han crecido observando la presencia de la computadora en la vida cotidiana, haciendo uso de éstas y de otros dispositivos que han utilizado para su disfrute y recreación; también han recibido la influencia de la apertura tecnológica, a través de los medios de comunicación, encargados de la divulgación de programas educativos y del arte de la Informática; adosado a este panorama, curiosamente existen textos escolares que traen anexo un disco compacto (CD) de contenido para provecho del estudiante, incluyendo actividades académicas para los niños, muchas veces producidos mediante juegos; entonces, ¿cómo esperar que este recurso sea debidamente aprovechado en la escuela?, si existe la posibilidad de que la maestra o maestro, profesora o profesor: (a) no haya alcanzado las competencias necesarias para sacar provecho a ese tipo de recurso; y/o (b) no tengan oportunidades de acceso al uso del computador. Lo anterior nos lleva a pensar críticamente acerca de lo siguiente:

¿Será que los docentes, durante su formación, no están sacando el máximo provecho al aprender sobre el computador y su uso en el aula de clases?, tan importante debe ser aprender sobre estrategias de enseñanza-aprendizaje como del uso del computador, puesto que el docente debe validar al computador como una herramienta que da soporte a su trabajo. Es así como el computador debe ser valorado como un recurso de mucho provecho para el docente.

Entonces, el personal que se está formando para ejercer la profesión docente debe prepararse para el uso de las tecnologías de la información y la comunicación, no de forma teórica, sino más bien, de forma práctica. Es preciso que existan, en los institutos y Universidades donde se imparte la carrera docente, laboratorios de computación en donde se puedan poner en práctica las potencialidades de las TIC, en apoyo a esta profesión; de tal forma que el futuro docente se ponga en contacto con la computadora, logre conocer su funcionamiento y las partes que la constituyen, así como su aplicación en la educación.

Cuando comento acerca de conocer el funcionamiento, no sólo me refiero a lo trivial: que la máquina es hardware que requiere un software básico de sistema que es el que evalúa el 
hardware disponible para poner en marcha el computador; sino, más bien se debe implementar software educativo que provenga tanto del Ministerio del Poder Popular para la Educación como de otras fuentes, siempre y cuando los contenidos estén acordes al nivel educativo donde se requieran utilizar; es decir, que el docente viva la experiencia de aprender a través del uso de programas cargados de elementos multimedia (sonido, texto, video, animaciones, hipertexto),los cuales causan un gran impacto visual y sonoro, haciendo más agradable el aprendizaje.

El uso de software educativo resulta beneficioso en las prácticas docentes, puesto que se traducen en insumos para enriquecer las explicaciones y demostraciones del docente hacia sus estudiantes, buscando en todo momento alcanzar mejores resultados en el proceso enseñanza-aprendizaje; y por otra parte, el uso de este tipo de software incentivará al docente a continuar en la búsqueda de estos programas, puesto que lo ayudan en su labor académica.

Hay que puntualizar que el software que se utilice debe estar desarrollado en apego a estándares de diseño gráfico, donde se utilicen colores y fuentes apropiados según el contenido presentado, considerando también las características de usuario potencial del mismo; además, sus contenidos deben presentarse de la manera más pedagógica posible, de forma que puedan brindar provecho en la enseñanza de temas o tópicos particulares.

Si no se aprovecha mejor el computador en la escuela, en el liceo, en la universidad o institutos de formación docente en Venezuela, entonces estaríamos contribuyendo a profundizar la brecha digital. Definida por Serrano y Martínez (2003), de la siguiente forma:

La separación que existe entre las personas (comunidades, estados, países...) que utilizan las Tecnologías de Información y Comunicación (TIC) como una parte rutinaria de su vida diaria y aquellas que no tienen acceso a las mismas y que aunque las tengan no saben cómo utilizarlas (p. 16).

Considerando lo anterior, se entiende el reto del Gobierno Nacional para buscar una mayor equidad en la sociedad, a través de las estrategias generadas para atender adecuadamente la incorporación de las TIC desde la escuela hasta las comunidades en general, proponiendo proyectos de apoyo a la infoestructura y otros en pro de la consolidación de la infocultura. Para ello se ha enfocado en la incorporación de las TIC en la propuesta curricular, en donde se trazan entre otros propósitos: forjar una ciudadanía que dignifique el uso de las tecnologías como factor de desarrollo humano y social; consolidar una nueva escuela bolivariana que mediante las TIC llegue a todos los escenarios de la vida republicana y en todos los momentos; aperturar espacios y/o canales para el flujo e intercambio de información veraz, vinculados con el proceso de enseñanza y aprendizaje; formar el potencial humano de la nueva escuela bolivariana en el uso y manejo adecuado de las TIC en razón del bien colectivo, conformar redes telemáticas de usuarios que fomenten el uso adecuado de las tecnologías en los procesos administrativos y académicos de la escuela. (Ministerio del Poder Popular para la Educación, 2010, p.15).

\section{Reconocimiento de experiencias en el uso de las TIC}

La Oficina Regional de Educación de UNESCO para América Latina y el Caribe (OREALC) desarrolló, durante el año 2004, un conjunto de estudios destinados a conocer 
experiencias destacadas de formación de profesores con incorporación de tecnologías de información y comunicación a propósito de aportar elementos clave en el diseño del currículo de formación inicial de los docentes. Los casos seleccionados corresponden a los países de Bolivia, Chile, Colombia, Ecuador, México, Panamá, Perú y Paraguay (OREALC, 2005, p.10). Es importante destacar que las experiencias seleccionadas para dicho estudio estuvieron conformadas por: programas o proyectos de formación probados en una institución establecida; con respaldo documental: objetivos, alcance, cobertura, metodología, mecanismos de monitoreo y evaluación, resultados de impacto, entre otros; aportes de nuevos conceptos y nuevas prácticas para el trabajo docente; duración considerable; capacidad de expansión a otras regiones; y que cuenten con investigaciones que sustenten su impacto positivo tanto en el trabajo docente como en el aprendizaje estudiantil. (p.12). De este documento se destacan algunas ideas para reflexionar, tales como:

Un docente que no maneje las tecnologías de información y comunicación está en clara desventaja con relación a los alumnos. La tecnología avanza en la vida cotidiana más rápido que en las escuelas, inclusive en zonas alejadas y pobres con servicios básicos deficitarios. Desafortunadamente, la sociedad moderna no ha sido capaz de imprimir el mismo ritmo a los cambios que ocurren en la educación.

Si bien todavía un importante número de escuelas no posee computadoras, proyector de imágenes o acceso a Internet, esto no necesariamente quiere decir que los estudiantes no estén siendo usuarios de juegos de video, aparatos de audio, Internet, telefonía celular, etc. En el campo de las tecnologías los estudiantes, de todas maneras, las aprenden y utilizan en otros contextos (p.9).

El citado documento recoge una serie de experiencias que, en materia de las TIC, se han indagado en los países seleccionados para el caso de estudio, donde, si bien los resultados no se destacan por tener grandes cantidades de proyectos en TIC, sí permiten apreciar que los que han emprendido arrojan buenos resultados; lo anterior da cabida a que otras naciones puedan conocer sus experiencias y puedan apoyarse en éstas como antecedentes para desarrollar nuevos mecanismos que impulsen el uso de las TIC por parte de los docentes.

Entre los puntos de conclusión del estudio, resulta relevante señalar que la formación del docente en el uso de las TIC deben considerar con mayor énfasis la modalidad a distancia y combinarla con la modalidad de estudio presencial en los casos que lo amerite, ya que, de esta manera, los docentes se involucrarán mucho mejor en las dinámicas de autoformación y formación permanente. Ello permitiría, además, brindar una mayor cobertura en la capacitación docente. Por otra parte hace alusión a la intervención de los Estados en fortalecer estos programas y atender también a la preparación de los tutores que se encargarán de materializar estos esfuerzos (2005, p. 183).

Llama poderosamente la atención, que en países como México se encuentran noticias donde en los laboratorios de computación, los docentes, sus estudiantes y otro voluntariado especialista en computación, trabajan en conjunto y buscan aprovechar al máximo estos recursos; elaborando estrategias de enseñanza y evaluando producciones digitales que puedan incorporar 
en sus clases. Además cuentan con portales Web cargados de contenido educativo, tanto para docentes como para los estudiantes, por ejemplo, portal edufuturo.com. Otra experiencia es la creación de programas de formación a distancia, tal es el caso de las actividades emprendidas por la Coordinación de Educación Virtual de la Universidad Autónoma Metropolitana Unidad Iztapalapa (UAMI), creada a partir del año 2009 con el objetivo de implementar la educación a distancia en los planes y programas de estudio, para propiciar el uso eficiente de las TIC en la práctica docente y en el diseño de metodologías y estrategias pedagógicas en modalidades no convencionales de enseñanza-aprendizaje. (UAMI, 2008).

Otro caso que ilustra estas experiencias, es la iniciativa en Argentina, donde poseen un portal Web dirigido a los docentes que quieran participar en actividades de actualización a distancia, tal como es el caso del portal educativo argentino, Ministerio de Educación de Argentina (2008).

En Colombia también se encuentran organismos orientados a la atención de la formación docente hacia el uso no sólo de la tecnología, sino también en otras áreas temáticas que le son de ayuda en su rol diario. Se puede mencionar por parte del Ministerio de Educación Nacional: (a) el Centro Virtual de Noticias de la Educación, en donde se publican noticias, convocatorias a concursos, cursos, simposios y todo tipo de actividades de índole académico en modalidades presenciales y virtuales (Ministerio de Educación Nacional de Colombia, 2012); (b) Colombia Aprende, portal Web activo desde el 2004, dirigido a docentes y directivos de básica, estudiantes de básica, familia y comunidad, estudiantes de educación superior, docentes y directivos de educación superior, e investigadores; ofrece acceso a objetos virtuales de aprendizaje e informativos (OVAI), además de noticias, proyectos, ofertas de formación en TIC, acceso a una red virtual de tutores, redes virtuales de aprendizaje, otros recursos y herramientas de interés académico (Ministerio de Educación Nacional de Colombia, 2004). Además se pudo consultar EDUTEKA, un portal educativo gratuito de la Fundación Gabriel Piedrahita Uribe (FGPU); se publica desde 2001 y se actualiza mensualmente. En este portal se pone a disposición centenares de contenidos formativos e informativos que les ayudan a enriquecer, con el uso de las TIC, los ambientes escolares de aprendizaje. Además, diseña e implementa espacios interactivos en línea con recursos útiles y valiosos para los docentes (Gestor de Proyectos de Aula, Planeador de Proyectos Colaborativos, Currículo Interactivo 2.0), entre otros elementos. Es importante resaltar que este portal Web ha recibido reconocimiento por parte del Ministerio de Educación Nacional, por la excelente calidad de la información que ofrece (Eduteka, 2000).

Por su parte, en Panamá destaca el Proyecto Entre Pares Panamá, a partir del cual se ha capacitado, en su primera fase, al 100\% de los docentes del país (35,300 docentes a nivel nacional) en el Uso Pedagógico de las Nuevas Tecnologías en Educación con la ayuda de 273 docentes líderes/facilitadores y 13 coordinadores regionales. La capacitación incluye 4 módulos: Alfabetización Digital, Herramientas de Comunicación e Internet, Comunidades de Aprendizaje/ Portales Educativos y Diseño de Proyectos de Integración de TIC. Los docentes, luego de la capacitación de 2 semanas, han podido implementar Proyectos de Integración de las TIC en sus aulas para facilitar el aprendizaje de los estudiantes y han adquirido una laptop como herramienta de trabajo, a un costo representativo de B/.100.00. Los docentes líderes también han obtenido reconocimientos y premiaciones a nivel internacional por sus proyectos de aula. La segunda fase del proyecto contempla capacitaciones presenciales y a 
distancia, y un acompañamiento/seguimiento a los docentes en sus aulas de clase por medio de visitas presenciales (Meduca, 2013).

Además encontramos el proyecto Educa Panamá, un portal Web del Ministerio de Educación de Panamá, diseñado estratégicamente para la innovación, creatividad y actualización tecnológica de la comunidad en general; busca apoyar el desarrollo de las actividades académicas, culturales, científicas y tecnológicas, así como la integración de la comunidad educativa a los espacios colaborativos, para el intercambio de experiencias que enriquezcan el proceso de enseñanza aprendizaje. Este portal forma parte de la Red Latinoamericana de Portales Educativos (RELPE), conformada por dieciocho (18) países (Educa Panamá, 2010).

En Venezuela, existe la Red Nacional de Actualización Docente mediante la Informática y la Telemática (RENADIT), cuyo objetivo expresa lo siguiente:

Nuestro objetivo fundamental es conformar una red humana de docentes, instituciones educativas y especialistas de las diferentes áreas del conocimiento para la articulación de esfuerzos, que impulsen el uso de las Tecnologías de la Información y la Comunicación (TIC), en el proceso de enseñanza y aprendizaje con la finalidad de elevar la calidad del sistema educativo venezolano. (RELPE, s.f.).

RENADIT cuenta con un portal Web, donde el docente Venezolano puede documentarse a través de artículos de interés; también se pueden descargar videos educativos, además de ofrecer la opción, al visitante, de inscribirse en los distintos cursos a distancias que tengan a bien ofertar desde el Ministerio del Poder Popular para la Educación. Por otra parte, en este entorno también se suministra información relevante acerca de disposiciones legales en torno a las TIC, manuales de referencia a docentes, diccionarios en línea, entre otras producciones.

Lo anterior apuntala a que en nuestro país también se han hecho esfuerzos importantes para la formación y actualización docente; sin embargo, considero que estas intervenciones no han sido evaluadas en función de su impacto en la educación, a fin de vigilar y garantizar su significancia y aporte al proceso enseñanza aprendizaje; al respecto puedo comentar por ejemplo, que el portal mencionado contaba, para el momento de la consulta, con veintidós (22) actividades de aprendizaje computarizadas, ocho (8) software educativos, varios micros, manuales, mapas, que constituyen producciones disponibles para ser descargados por los docentes u otros usuarios visitantes. Estas cifras indudablemente deben elevarse a razón de poner a disposición mayor cantidad de recursos por cada nivel educativo.

Por otra parte, sería ideal que se incrementaran sitios de este propósito para dar asistencia a los docentes venezolanos; sitios que estén administrados por personal vinculado a la educación, altamente comprometidos en mantener novedades que impacten y promuevan el espíritu investigador de los docentes; ofreciendo apoyo a estos profesionales, que de seguro acudirán a estos portales educativos confiados de encontrar día a día, información ajustada a sus necesidades.

Otro ejemplo a citar es el Plan Nacional de Alfabetización Tecnológica (PNAT), creado en el año 2001 por el Ministerio del Poder Popular para Ciencia, Tecnología e Industrias Intermedias (MCTI), a través de la Misión Ciencia. El objetivo del PNAT es propiciar espacios de aprendizaje para los ciudadanos y ciudadanas, proporcionándoles herramientas 
que les permitan el acceso a la información a través de las TIC; se ofrece enseñanza para el uso del computador mediante un curso gratuito, compuesto por un conjunto de módulos de corta duración, impartido por voluntariado en las universidades y otros sitios estratégicos del país, como por ejemplo en los Infocentros. (MCTI, 2008).

El PNAT, lo ejecuta el Centro Nacional de Tecnologías de Información (CNTI) y comprende un curso básico de software libre en veinticuatro (24) horas, que consiste en capacitar a los participantes en el uso de la computadora, así como enseñarles a elaborar textos (usando procesador de palabras), gráficos, dibujos y crear, enviar y recibir correos electrónicos. Un curso bien planificado de veinticuatro (24) horas es suficiente para ese contenido; sin embargo debe existir la oportunidad de que estos ciudadanos que acuden a la inducción tecnológica tengan acceso a las computadoras para realizar sus prácticas sobre lo que aprendieron. Pudiera traducirse esto, en ampliar la extensión del curso a fin de permitir una práctica supervisada de los asistentes en cuanto a lo aprendido pare el debido uso de las TIC.

Sería de inmenso provecho que los docentes también puedan recibir asistencia para su preparación académica en el uso del computador, a través del PNAT; de aquí se desprende la idea de crear un módulo en el PNAT dedicado a la preparación del docente en la generación y evaluación de material de apoyo para el proceso enseñanza aprendizaje; es decir, una extensión del PNAT donde se incorpore al docente en el uso apropiado del computador como herramienta de trabajo en el salón de clases y para el uso personal; por ejemplo, que puedan utilizar el computador en conjunto con los portales Web gubernamentales para mantenerse en sintonía con los Proyectos Nacionales. Igualmente sería interesante que utilizaran los portales Web bancarios para hacer pagos, consultas en línea, etcétera, así como enterarse de las novedades que marcan la pauta hoy en día en el mundo, a través de las páginas de agencias de noticias.

Esta propuesta no escaparía de las manos del Estado, pues existe un contingente de estudiantes universitarios que está en el deber de cumplir lo que se denomina Servicio Comunitario, en correspondencia a lo dispuesto por la Ley de Servicio Comunitario del Estudiante de Educación Superior (2005), donde en su Artículo 4 refiere lo siguiente:

A los efectos de esta Ley, se entiende por Servicio Comunitario, la actividad que deben desarrollar en las comunidades los estudiantes de educación superior que cursen estudios de formación profesional, aplicando los conocimientos científicos, técnicos, culturales, deportivos y humanísticos adquiridos durante su formación académica, en beneficio de la comunidad, para cooperar con su participación al cumplimiento de los fines del bienestar social, de acuerdo con lo establecido en la Constitución de la República Bolivariana de Venezuela y en esta Ley. (p. 1)

Por otra parte, en el Artículo 8 de la mencionada Ley se establece que el estudiante debe cumplir, como mínimo, ciento veinte (120) horas de servicio; este tiempo pudiera aprovecharse también para atender las necesidades de los maestros y maestras, profesores y profesoras de nuestro país, tendiendo apoyo tanto a aquellos que trabajan en instituciones dependientes del Estado como a quienes laboran para instituciones privadas, porque a final de cuentas todos atienden a lo más valioso de nuestro país, su niñez y juventud, quienes se preparan en manos de sus docentes para asumir, en su debido momento, las riendas que encaminarán el progresivo avance de nuestra Nación. 


\section{Consideraciones para mejorar la experiencia en el uso de las TIC}

El primer lugar, considero que debería constituirse una campaña a nivel Nacional para fortalecer la enseñanza a los docentes tanto en formación como en ejercicio, en materia de las TIC; de tal forma que los docentes puedan valorar su uso tanto a nivel teórico como práctico y desarrollen las competencias necesarias para utilizarlas. De esta manera podrán sacarle provecho como instrumentos de comunicación y como medios para desarrollar y reproducir material de apoyo al proceso educativo, con lo que se suma efectividad al proceso enseñanza- aprendizaje.

Es imperante que el docente identifique y compruebe cómo trabajar con el acceso a Internet; que conozca dónde hallar recursos multimedia de contenido académico y significativo para su clase o temática de estudio, a fin de que el mismo tenga la capacidad y responsabilidad de evaluarlos previo a su uso.

En ese mismo orden de ideas, en la sociedad de conocimiento, donde la información es uno de los valores más importantes a conservar, es trascendental tanto manejar el computador, el acceso a Internet, como el uso debido y racional al cúmulo gigantesco de conocimientos que se pueden hallar en la red de redes. A través de la cual se pueden acceder a millones de contenidos o informaciones, pero que no deben ser consideradas como verdades a primera vista, sino que más bien deben aplicarse criterios para seleccionar la validez de la información encontrada en la Web, dado que no todo lo que se encuentra es necesariamente confiable o fidedigno. Es así como el docente debe distinguir entre un sitio personal y uno institucional y a su vez, aplicar criterios de selección de la información consultada, que apunten a escoger aquella que esté bien fundamentada, con referencias, con buena estructura de presentación y contenidos que puedan ser usados como recurso en el salón de clases.

Indudablemente, el rol docente tiene un gran desafío con la implementación de las TIC; por lo tanto es un tema importante a considerar para quienes tienen en sus manos la alta responsabilidad de adecuar los planes de estudios de las carreras donde se forman docentes; y también para quienes, entre sus responsabilidades, tienen establecer los mecanismos adecuados para que el personal docente en ejercicio se actualice en conocimientos y herramientas tecnológicas para el trabajo pedagógico, de tal forma que éstos puedan secundar las acciones que en materia tecnológica emprenda el Gobierno Nacional para favorecer la educación.

En suma, las TIC forman parte del quehacer diario, en cualquier profesión, más aún en la Educación; hacia allá se debe redirigir el proceso, es decir, formar al docente para que éste desarrolle las competencias en el aprovechamiento del computador, para que conozca cómo defenderse ante amenazas de virus, cómo proteger su información, cómo escoger los contenidos adecuados. Es más bien, enseñar al docente a administrar el computador, que es todo lo opuesto al simple curso de herramientas ofimáticas, tales como procesadores de texto y hojas de cálculo que se imparte a nuestros docentes y estudiantes.

¿Cómo encaminar este cambio?

1. Debe iniciar por mejorar lo que se tiene, es decir, dejar de sub utilizar los laboratorios de computación, comenzando por reestructurar su modelo operativo y organizativo; ampliando el personal que lo dirige y mantiene en funcionamiento; es decir, debe constituirse un cuerpo de profesores y estudiantes que tenga responsabilidades 
puntuales inherentes a la administración de tan preciado recurso. Eso implica cambiar el paradigma que se tiene en muchos laboratorios que cuentan con un profesor de la clase de computación, que es el mismo que lo administra para mantenerlo en funcionamiento. Con ese cambio, se podrá convertir en un sitio más productivo, donde se desarrolle software de provecho para fines didácticos y se comparta información.

2. Las autoridades universitarias a cargo del servicio comunitario deben promover alianzas con otras instituciones educativas, a fin de brindar información acerca de los planes de formación de docentes para el uso de las TIC. En éstos deben figurar: el tiempo programado para los cursos; cantidad de estudiantes facilitadores; cantidad de estudiantes (docentes en formación y/o ejercicio) participantes del curso; lugar de encuentro, puede tratarse de los laboratorios propios de las instituciones educativas, laboratorios de las universidades, laboratorios Alma Mater, CBIT, Infocentros o cualquier laboratorio institucional que esté presto a facilitar la localización del curso. Además debe informar sobre los contenidos pautados, entre los cuales se recogen temas como: conociendo al computador, internet, correo electrónico, gobierno electrónico, portal web del Ministerio del Poder Popular para la Educación, enlace a RENADIT; visitas a portales web de universidades que cuentan con enseñanza virtual, entre otros sitios de interés; uso de software para preparar presentaciones incorporando elementos como texto, imágenes, videos, sonido, hipertexto y otros enlaces; metodología para el diseño de material educativo, contemplando los estándares que deben incorporarse a esta labor de producción; evaluación de software educativo, alternativas disponibles para la publicación en la web de software educativo producido en los cursos.

3. Las autoridades universitarias a cargo del servicio comunitario deben promover alianzas los sitios disponibles para realizar los cursos, concretando toda una serie de normas que regirán la cooperación entre las partes y donde se pongan de manifiesto los procedimientos que garanticen el buen uso de los equipos e instalaciones a utilizar en el marco de la ejecución de convenios para la formación de docentes en el uso de las TIC; de igual forma deben convenir acerca de la manera en que serán otorgados los recursos necesarios para cumplir con la entrega de certificaciones a los docentes.

4. Las autoridades universitarias a cargo del servicio comunitario ofertarán las actividades planificadas y velarán por su correcta ejecución, manteniendo documentación de lo acontecido, logros, observaciones, ajustes a realizar, entre otros aspectos propios de considerar. En base a lo anterior, construir un sistema de información que pueda ser consultado para evaluar el impacto de la intervención de los estudiantes universitarios en la formación de docentes para el uso de las TIC.

5. Las instituciones educativas deben facilitar la intervención de sus docentes a los cursos de formación, lo que significa que debe existir una planificación de contingencia para suplir al personal que asistirá a las sesiones pautadas para los cursos; de esta forma no se deberá afectar el normal desenvolvimiento de las actividades escolares.

6. Se deben realizar evaluaciones semestrales al resultado de esta experiencia de intercambio de conocimientos, a fin de obtener información que sirva para realimentar este trabajo colaborativo. Las evaluaciones deben originarse en las 
instituciones educativas, quienes indagarán acerca de la efectividad del curso para sus docentes; y por parte de las universidades, deben indagar acerca de lo que sus estudiantes exponen en su informe final de servicio comunitario, donde plasman sus experiencias y sugerencias al proceso donde ejecutaron su prestación.

Tal como se puede observar, se puntualizan algunos aspectos que engloban el proceso de intercambio y colaboración entre universidades y otras instituciones educativas; la operatividad del mismo dependerá del grado de empatía que se tenga con esta idea de trabajo colaborativo, donde ambas partes buscan un beneficio que se traduce en aumentar, por parte de los estudiantes universitarios, sus cualidades de apoyar a sus semejantes al logro de objetivos concretos y por parte de los docentes, alcanzar mayor dominio en el uso de las TIC.

\section{Consideraciones Finales}

En Latinoamérica se puede palpar, a través de las noticias publicadas en la red de redes, los esfuerzos que desde cada país se hacen para abordar en mayor cantidad y calidad el tema del uso de las TIC en la escuela, Lo anterior representa un arduo trabajo que no termina, puesto que las tecnologías cambian, se expanden y dinamizan el entorno educativo, que debe mantenerse al día para dejar atrás la brecha digital.

Por su parte, el estado venezolano ha concretado esfuerzos para llevar la tecnología a los centros de estudios para que estén a disposición de todos; del mismo modo, ha consolidado una red de actualización docente en el uso de las TIC, la cual se debe fortalecer enormemente para dar atención a, maestras, maestros, profesoras y profesores en cuanto a su inclusión al uso de esta tecnología, valiéndose para ello del potencial que representa el servicio comunitario. La idea es que, de manera expedita, se puedan poner, en manos del docente, las herramientas que necesita para emprender trabajos de investigación, de desarrollo personal y para la producción de material de apoyo didáctico de calidad para utilizarlo en el aula de clases, utilizando para ello, el PNAT como proyecto pedagógico, y a los prestadores de servicio comunitario como mano ejecutora del mismo.

Es válido suponer que la ausencia del conocimiento técnico, para utilizar el computador al servicio de la educación, ha representado obstáculos a los docentes, puesto que eso se puede traducir en limitaciones tanto personales como profesionales, dado que implica la dependencia hacia terceras personas para llevar a cabo alguna tarea que forzosamente amerite el uso del computador. Para contrarrestar esta dependencia, la idea de articular el Programa PNATServicio Comunitario resulta una estrategia viable que se orienta a apoyar el mejor uso de las TIC por parte de los docentes en formación.

El aprovechamiento de los proyectos, que en materia Informática el gobierno ha dispuesto, debe elevarse en cantidad y calidad, lo cual se corresponde con una idea de revisión del impacto de éstos a nivel nacional, pudiéndose encontrar que, entre la variedad de estrategias trazadas en la Nación, se puedan estrechar vínculos y establecer trabajos en conjunto, como es el ejemplo citado de aprovechamiento del contingente estudiantil que presta servicio comunitario, para incorporarlos como voluntarios al Plan Nacional de Alfabetización Tecnológica (PNAT); de esta manera se propician espacios donde se encamine 
hacia la especialización de los cursos impartidos a través del PNAT para que se ajusten a las necesidades del docente en formación y en ejercicio.

\section{Referencias}

Cardona, N. (2004). Renadit. Infobit: Revista para la difusión y el uso educativo de las tecnologías de la información y la comunicación (3). p.16-18. Recuperado de: http:// fundabit.gob.ve/descargas/revistas/Edicion-03.pdf

Educa Panamá. (2010). Mi portal Educativo. Panamá: Ministerio de Educación. Recuperado de: http://www.educapanama.edu.pa/

Eduteka.org (2000). Quiénes somos. Recuperado de: http://www.eduteka.org/

Fundabit. (s.f.). Proyectos. Venezuela: Fundación Bolivariana de Informática y Telemática. Recuperado de: http://fundabit.me.gob.ve/proyectos/

Henríquez, M. (2004). La incorporación de las tecnologías de la información en la formación inicial docente. Caso de estudio: Universidad de los Andes, Táchira. Revista Acción Pedagógica, 11(1), pp 60-73. Recuperado de: http://www.saber.ula.ve/ bitstream/123456789/17067/1/art6_v11n1.pdfhttp://www.saber.ula.ve/db/ssaber/Edocs/ pubelectronicas/accionpedagogica/vol11num1/art6_v11n1.pdf

Ley de Servicio Comunitario del Estudiante de Educación Superior. (14 de septiembre de 2005). Gaceta Oficial de la República Bolivariana de Venezuela, $N^{\circ} 38.272$. Recuperado de http://es.slideshare.net/HumanitasFHE/ley-de-servicio-comunitario-del-estudiantede-educacin-superior- 4538606

MCTI. (2008). Portal del Ministerio del Poder Popular para Ciencia, Tecnología e Industrias Intermedias. Venezuela. Recuperado de: http://www.mppcti.gob.ve/

Meduca (2013). Meduca para toda la vida. Panamá: Ministerio de Educación. Recuperado de: http://www.meduca.gob.pa/files/Revista\%20MEDUCA\%202013.pdf

Ministerio de Educación de Argentina (2008). Educar Portal Argentino para Capacitación Docente a Distancia. Recuperado de: http://portal.educ.ar/capacitacion/

Ministerio de Educación Nacional de Colombia. (2004). Colombia Aprende. Recuperado de: http://www.colombiaaprende.edu.co/html/directivos/1598/propertyvalue-34418.html 
Ministerio de Educación Nacional de Colombia. (2012). Centro Virtual de Noticias de la Educación de la República de Colombia. Recuperado de: http://www.mineducacion.gov. co/cvn/1665/w3-channel.html.

Ministerio del Poder Popular para la Educación (s.f.). Proyecto Canaima Educativo: corazón y esfuerzo venezolano. Recuperado de: http://www.me.gob.ve/media/ contenidos/2012/d_26076_308.pdf

Ministerio del Poder Popular para la Educación. (2010). Líneas Generales para la Incorporación de las Tecnologías de la Información y la Comunicación en los Procesos de Aprendizaje enmarcadas en la Propuesta Curricular de la República Bolivariana de Venezuela (20072013). Recuperado de: http://www.me.gob.ve/media/contenidos/2011/d_26034_245.pdf

Miratia, O. (2005). Las Tecnologías de la Información y la Comunicación en la Educación. Revista Infobit (4), pp. 12-14. Recuperado de: http://fundabit.gob.ve/descargas/revistas/ Edicion-04.pdf

OREALC. (2005). Informe: Formación docente y las tecnologías de información y comunicación. Logros, tensiones y desafíos. Chile: Oficina Regional de Educación de la UNESCO para América Latina y el Caribe. Recuperado de: http://unesdoc.unesco.org/ images/0014/001410/141010s.pdf

PNUD (2002). Informe sobre Desarrollo Humano en Venezuela 2002. Las tecnologías de la información y comunicación al servicio del desarrollo. Venezuela: Programa de las Naciones Unidas para el Desarrollo. Recuperado de: http://hdr.undp.org/sites/ default/files/venezuela_2002_es.pdfhttp://www.undp.org/annualreports/2002/espanol/ IAR02spaComplete.pdf

RELPE (s.f.). El portal educativo de Venezuela presenta Renadit. Red Latinoamericana de Portales Educativos. Recuperado de: http://www.relpe.org/ultimasnoticias/el-portaleducativo-de-venezuela-presenta-renadit/

Serrano, A., Martínez, E. (2003). La Brecha Digital: Mitos y Realidades. México: Departamento Editorial Universitaria, Universidad Autónoma de Baja California. Recuperado de: http://labrechadigital.org/labrecha/LaBrechaDigital_MitosyRealidades. pdfhttp://labrechadigital.com

UAMI. (2008). Programa de Formación Docente de la Universidad Autónoma Metropolitana Unidad Iztapalapa. Recuperado de: http://virtuami.izt.uam.mx/pfd/ 